University of Wollongong

Research Online

Faculty of Social Sciences - Papers (Archive) Faculty of Arts, Social Sciences \& Humanities

$1-1-2020$

Wholegrain and legume consumption and the 5-year incidence of agerelated cataract in the Blue Mountains Eye Study

Ava Tan

Victoria Flood

Annette Kifley

Joanna Russell

University of Wollongong, jrussell@uow.edu.au

Robert Cumming

See next page for additional authors

Follow this and additional works at: https://ro.uow.edu.au/sspapers

Part of the Education Commons, and the Social and Behavioral Sciences Commons

Research Online is the open access institutional repository for the University of Wollongong. For further information contact the UOW Library: research-pubs@uow.edu.au 


\title{
Wholegrain and legume consumption and the 5-year incidence of age-related cataract in the Blue Mountains Eye Study
}

\author{
Abstract \\ The Authors 2020. The present study aims to investigate the effect of wholegrain and legume \\ consumption on the incidence of age-related cataract in an older Australian population-based cohort. The \\ Blue Mountains Eye Study (BMES) is a population-based cohort study of eye diseases among older adults \\ aged 49 years or older (1992-1994, $n=3654)$. Of 2334 participants of the second examination of the BMES \\ (BMES 2, 1997-2000), 1541 (78.3\% of survivors) were examined 5 years later (BMES 3 ) who had \\ wholegrain and legume consumption estimated from the food frequency questionnaire (FFQ) at BMES 2. \\ Cataract was assessed using photos taken during examinations following the Wisconsin Cataract \\ Grading System. Multivariable-Adjusted logistic regression models were used to assess associations with \\ the 5-year incidence of cataract from BMES 2 (baseline) to BMES 3. The 5-year incidence of cortical, \\ nuclear and posterior subcapsular (PSC) cataract were $18.2 \%, 16.5 \%$ and $5.9 \%$, respectively. After \\ adjustment for age, sex and other factors, total wholegrain consumption at baseline was not associated \\ with incidence of any type of cataract. High consumption of legume showed a protective association for \\ incident PSC cataract (5th quintile: Adjusted odds ratio 0.37, 95\% confidence interval 0.15-0.92). There \\ was no significant trend of this association across quintiles $(P=0.08)$. In this older Australian population, \\ we found no associations between wholegrain intake at baseline and the 5-year incidence of three \\ cataract types. However, intake of legumes in the highest quintile, compared to the lowest quintile, may \\ protect against PSC formation, a finding needing replication in other studies.

\section{Disciplines} \\ Education | Social and Behavioral Sciences

\section{Publication Details} \\ Tan, A., Flood, V., Kifley, A., Russell, J., Cumming, R., Mitchell, P. \& Wang, J. (2020). Wholegrain and legume \\ consumption and the 5-year incidence of age-related cataract in the Blue Mountains Eye Study. British \\ Journal of Nutrition,

\section{Authors} \\ Ava Tan, Victoria Flood, Annette Kifley, Joanna Russell, Robert Cumming, Paul Mitchell, and Jie Wang
}




\title{
Wholegrain and legume consumption and the 5-year incidence of age-related cataract in the Blue Mountains Eye Study
}

\author{
Ava Grace Tan ${ }^{1}$, Victoria M. Flood ${ }^{2,3}$, Annette Kifley ${ }^{1}$, Joanna Russell ${ }^{4}$, Robert G. Cumming ${ }^{5}$, Paul Mitchell ${ }^{1}$ \\ and Jie Jin Wang ${ }^{1,6 *}$ \\ ${ }^{1}$ Centre for Vision Research, Department of Ophthalmology, The Westmead Institute for Medical Research, The University of \\ Sydney, Sydney, NSW, Australia \\ ${ }^{2}$ Faculty of Health Sciences, The University of Sydney, Sydney, NSW, Australia \\ ${ }^{3}$ Western Sydney Local Health District, Westmead Hospital, Westmead, NSW, Australia \\ ${ }^{4}$ School of Health \& Society, Faculty of Social Sciences, University of Wollongong, Wollongong, NSW, Australia \\ ${ }^{5}$ School of Public Health, The University of Sydney, Sydney, NSW, Australia \\ ${ }^{6}$ Health Services and Systems Research, Duke-NUS Medical School, Singapore, Singapore \\ (Submitted 5 March 2019 - Final revision received 8 March 2020 - Accepted 12 March 2020)
}

\section{Abstract}

The present study aims to investigate the effect of wholegrain and legume consumption on the incidence of age-related cataract in an older Australian population-based cohort. The Blue Mountains Eye Study (BMES) is a population-based cohort study of eye diseases among older adults aged 49 years or older (1992-1994, $n$ 3654). Of 2334 participants of the second examination of the BMES (BMES 2, 1997-2000), 1541 (78.3\% of survivors) were examined 5 years later (BMES 3 ) who had wholegrain and legume consumption estimated from the FFQ at BMES 2. Cataract was assessed using photographs taken during examinations following the Wisconsin cataract grading system. Multivariable-adjusted logistic regression models were used to assess associations with the 5-year incidence of cataract from BMES 2 (baseline) to BMES 3. The 5-year incidence of cortical, nuclear and posterior subcapsular (PSC) cataract was 18.2, 16.5 and 5.9\%, respectively. After adjustment for age, sex and other factors, total wholegrain consumption at baseline was not associated with incidence of any type of cataract. High consumption of legumes showed a protective association for incident PSC cataract (5th quintile: adjusted OR 0.37;95\% CI 0.15, 0.92). There was no significant trend of this association across quintiles $(P=0 \cdot 08)$. In this older Australian population, we found no associations between wholegrain intake at baseline and the 5-year incidence of three cataract types. However, intake of legumes in the highest quintile, compared with the lowest quintile, may protect against PSC formation, a finding needing replication in other studies.

Key words: Age-related cataracts: Whole grains: Legumes: Epidemiology

Age-related cataract is the opacification of the eye lens occurring from the age of 50 years onwards ${ }^{(1,2)}$ and remains a major cause of vision impairment worldwide ${ }^{(3)}$. There are three types of age-related cataract, each affecting a different part of the lens: cortical, nuclear and posterior subcapsular (PSC) cataract $^{(1,2)}$. Strategies to delay development and slow progression of age-related cataract would reduce eye health care cost and the burden on affected persons and their families. Nutritional and dietary modification is an attractive preventive strategy from a public health standpoint. As such, there has been much interest in dietary factors that may affect cataractogenesis. While individual nutrients have shown some beneficial effect on cataract formation $^{(4-7)}$, individual nutrients are rarely consumed in isolation. Moreover, nutrient supplements have not shown the same benefits in clinical trials as that observed in observational studies $^{(8)}$. It may be more beneficial and practical to investigate key components of the diet, rather than individual nutrients, to further elucidate the benefit of diet on eye and systemic health.

Both whole grains and legumes are slow-release carbohydrates, rich in fibre, good sources of antioxidants and have a lower glycaemic index $(\mathrm{GI})^{(9-11)}$. Higher intakes of whole grains have been shown to have beneficial effects for diabetes, CVD, obesity and mortality ${ }^{(12-14)}$. Similarly, legume consumption has also been shown to be associated with lower risks of CVD $^{(15)}$, diabetes ${ }^{(16)}$ and mortality ${ }^{(17)}$. However, there is limited

Abbreviations: BMES, Blue Mountains Eye Study; GI, glycaemic index; PSC, posterior subcapsular.

* Corresponding author: Jie Jin Wang, email jiejin.wang@duke-nus.edu.sg 
information on the association between wholegrain and legume consumption and the risk of age-related cataract.

Due to the high nutritional content and antioxidant properties of whole grains and legumes, we speculate that high consumption of whole grains and/or legumes by older people may have protective effects on the eye and/or ageing, as nuclear cataract is considered an ageing marker. In this report, we aimed to investigate the longitudinal associations between wholegrain and legume consumption at baseline and the 5-year incidence of age-related cataract in an older Australian cohort sample, the Blue Mountains Eye Study (BMES) population.

\section{Methods \\ Study population}

The BMES is an observational, population-based cohort study of vision and common eye diseases in a population aged 49 years and older, living in the Blue Mountains region, west of Sydney, Australia. The baseline examinations (BMES 1, 1992-1994) were conducted on 3654 participants ( $82.4 \%$ of eligible). Five years later (1997-1999, BMES 2), 2334 (75.1\% of survivors) participants were re-examined and 1952 (76.6\% of survivors) participants were followed 10 (2002-2004, BMES 3) years later. For this report, the BMES 2 population serves as the baseline of this cohort, where both wholegrain and legume consumption information was available, and the 5-year incidence of cataract was assessed using lens photographs taken at BMES 2 and BMES 3 examinations.

\section{Examination procedures}

Detailed examination procedures have been described previously $^{(18)}$. The same procedures were conducted for all examinations. Briefly, after pupil dilation, each participant underwent a detailed eye examination, including lens photography. Interviewer-administered questionnaires were used to collect demographic, dietary data and medical information. Written, informed consent was obtained from each participant. The study adhered to the tenets of the Declaration of Helsinki and was approved by the Human Ethics Committees of the University of Sydney and the Western Sydney Area Health Service.

\section{Cataract grading}

Lens photographic and cataract grading procedures have been described in detail previously ${ }^{(18)}$. In brief, lens photographs taken during eye examinations were assessed for cataract in a masked manner following the Wisconsin cataract grading system $^{(19)}$. Retroillumination (Neitz CT-R; Neitz Instruments) and slit lamp (Topcon SL-7 E, Topcon Optical Co.) lens photographs taken during the eye examinations were used to assess cataract. Cortical and PSC cataract were assessed using a grid overlay to allow estimation of the percentage area of involvement for each type of cataract. Cortical cataract was defined as $\geq 5 \%$ of total area involved. PSC cataract was defined as any such opacity present. Nuclear cataract was assessed by comparing the slit lamp photograph to a set of four standard photographs and was defined as nuclear opacity greater than standard photograph no. 3. Inter- and intragrader reliability of the cataract grading have been reported previously ${ }^{(20)}$.

A participant without a particular type of cataract in any eye at baseline was considered at risk of developing that particular type of cataract in the follow-up and was included in the assessment of incidence of this cataract type. For example, if a participant had nuclear cataract only at baseline, they would be considered at risk of developing both cortical and PSC cataract at follow-up.

\section{Dietary assessment}

Participants were also asked to complete a 145 -item semiquantitative FFQ to assess typical food consumption over the past year. The FFQ was a modified Willett $\mathrm{FFQ}^{(21)}$ for the Australian diet and vernacular and included portion size estimates and frequency. Brand details of breakfast cereals were also collected. Consumption frequency was measured using nine categories from 0 to 4 serves/d. Responses to each FFQ item were then converted to daily intake. Daily nutrient intakes were estimated using purpose-built software incorporating the Australian Tables of Food Composition (NUTTAB95) ${ }^{(22)}$. Validation of the FFQ was conducted in a subsample of the population ( $n$ 78) and compared against the average of three, 4-d weighed food records over 12 months ${ }^{(21)}$.

We estimated wholegrain consumption frequency and daily intake from four items in the FFQ: breakfast cereal, oatmeal, wholemeal/multigrain breads and brown rice. Estimates of wholegrain intakes in $\mathrm{g} / \mathrm{d}$ were calculated by multiplying the wholegrain percentage of each wholegrain food, the weight per serve of wholegrain food, determined from NUTTAB95(22), and the number of serves/d indicated in the FFQ. Wholegrain percentage of breakfast cereal items was based on nutritional information available from the food company or manufacturers' website or estimated from similar breakfast cereals in the absence of manufacturer information as determined by the study dietitian (V. M. F.). Wholegrain percentage of oatmeal, wholemeal/multigrain breads and brown rice items was based on values reported in The Grains \& Legumes Health Report ${ }^{(23)}$.

Legume consumption was determined using three items from the FFQ: baked beans, soyabeans, and other beans and lentils. Estimates of legume intake in $\mathrm{g} / \mathrm{d}$ were calculated by multiplying the weight per serve of legumes, determined from NUTTAB95 ${ }^{(22)}$, by the number of serves/d indicated in the FFQ.

Wholegrain, legume and other nutrient intakes were energyadjusted following the residual method described by Willett \& Stampfer ${ }^{(24)}$. Total diet score was developed for BMES ${ }^{(25)}$ to assess adherence to Australian dietary guidelines with higher scores indicating higher adherence to dietary recommendations. One serve of wholegrain food is estimated as having $8 \mathrm{~g}$ wholegrain intake, in line with Australian dietary guidelines ${ }^{(26,27)}$, where six serves are equivalent to the daily target intake of $48 \mathrm{~g}$ whole grains. One serve of legume food is estimated as having $75 \mathrm{~g}$ legume intake, also in line with Australian dietary guidelines $^{(27,28)}$. 


\section{Statistical analysis}

All analyses were conducted using SAS 9.4 (SAS Institute). Baseline characteristics were compared for BMES 2 participants who did and did not attend the follow-up examination 5 years later (Table 1). Differences in baseline characteristics were tested using independent $t$ test for continuous variables (mean values and standard deviations) and Pearson's $\chi^{2}$ test for categorical variables (frequencies in percentages). Quintiles of wholegrain and legume intake were created with the lowest quintile as the reference group. Characteristics of participants attending the follow-up visits were compared across quintiles of wholegrain and legume consumption (Table 2). Differences across quintiles were tested using Mantel-Haenszel $\chi^{2}$ test.

Potential confounding variables at baseline of this report (BMES 2) included age, sex, smoking status (past, current and never smokers), hypertension (defined as systolic blood pressure $\geq 160 \mathrm{mmHg}$, diastolic blood pressure $\geq 100 \mathrm{mmHg}$, self-reported hypertension or use of antihypertensive medications), diabetes (defined as fasting blood glucose $\geq 7.0 \mathrm{mmol} / \mathrm{l}$ or self-reported diabetes or use of diabetic medication), education (attainment of trade certificate or higher degree), self-reported use of inhaled or oral steroids and the presence of myopia (spherical equivalent of $<-1 \cdot 0$ dioptre). The following potential cofounding nutritional variables associated with the exposures or the outcomes were also considered: total diet score, energyadjusted fibre, vitamin $\mathrm{C}$, vitamin $\mathrm{E}, \mathrm{Zn}$, lutein, zeaxanthin and beta-carotene. Because different cataract types may be associated with different risk factors, the final parsimonious models varied for each cataract type and only included variables that were significantly $(P<0.05)$ associated with that particular cataract type, as determined by stepwise regression process.
Multivariable-adjusted logistic regression models were used to assess the associations between baseline (at BMES 2) wholegrain and legume consumption (by quintiles and per serve/d increase) and 5-year cataract incidence. OR and $95 \% \mathrm{CI}$ are presented. $P_{\text {for trend }}$ was calculated using the median trend method. Statistical significance was set at $P<0 \cdot 05$.

\section{Results}

Of the 2334 participants in BMES 2, 1722 (87.4\% of survivors) returned for the BMES 3 examinations. Those who participated in BMES 2 but not BMES 3 were older, more likely to be male, a current smoker or have greater pack/years smoked, a history of hypertension, have prevalent cortical and nuclear cataract and lower consumption of whole grains and vitamin $\mathrm{E}$ but less likely to have higher education than those who did participate in the BMES 3 examinations (Table 1).

Of the 1722 participants, 1541 (89.5\%) had usable FFQ with estimated wholegrain and legume consumption. The mean intake of whole grains in this population was 49.6 (sD 34.8) g/d, with $49.2 \%$ of the population consuming the recommended daily target intake of $48 \mathrm{~g}$. The mean legume intake was 18.5 (SD 29.9) g/d. Table 2 presents the baseline characteristics of the entire BMES 2 population by quintiles of energy-adjusted wholegrain and legume consumption. Participants with higher intakes of whole grains were less likely to be current smokers or have less pack/years smoked, also less likely to have hypertension and high BMI, but more likely to have higher education and higher dietary fibre and vitamin E intake (Table 2). Participants with higher intakes of legumes were younger, more likely to be female, to have hypertension and higher dietary fibre intake

Table 1. Comparison of characteristics of the Blue Mountains Eye Study 2 participants and non-participants of the follow-up examination 5 years later (Mean values and standard deviations; numbers and percentages)

\begin{tabular}{|c|c|c|c|c|c|c|c|}
\hline \multirow[b]{2}{*}{ Baseline characteristics } & \multicolumn{3}{|c|}{ Participants (n 1722) } & \multicolumn{3}{|c|}{ Non-participants (n 612) } & \multirow[b]{2}{*}{$P+$} \\
\hline & Mean & $\%$ & SD & Mean & $\%$ & SD & \\
\hline Age (years) & 68.0 & & $7 \cdot 8$ & $74 \cdot 2$ & & 9.5 & $<0.001 \ddagger$ \\
\hline Female & & $59 \cdot 1$ & & & $53 \cdot 1$ & & $<0.001 \ddagger$ \\
\hline Non-smoker & & $52 \cdot 0$ & & & 44.9 & & $<0.001 \ddagger$ \\
\hline Past-smoker & & $40 \cdot 2$ & & & $42 \cdot 9$ & & \\
\hline Current smoker & & $7 \cdot 8$ & & & $12 \cdot 2$ & & \\
\hline Pack/years of smoking & $12 \cdot 4$ & & $23 \cdot 3$ & $16 \cdot 6$ & & $28 \cdot 3$ & $0.001 \ddagger$ \\
\hline Hypertension & & $51 \cdot 7$ & & & $59 \cdot 1$ & & $0.002 \ddagger$ \\
\hline Diabetes & & $10 \cdot 0$ & & & $12 \cdot 8$ & & 0.06 \\
\hline Education§ & & $62 \cdot 5$ & & & 54.4 & & $<0.001 \ddagger$ \\
\hline Myopia & & $14 \cdot 6$ & & & $17 \cdot 1$ & & 0.16 \\
\hline Inhaled steroids & & $15 \cdot 0$ & & & $14 \cdot 8$ & & 0.91 \\
\hline Cortical cataract & & $24 \cdot 2$ & & & $36 \cdot 0$ & & $<0.001 \ddagger$ \\
\hline Nuclear cataract & & $26 \cdot 8$ & & & $44 \cdot 6$ & & $<0.001 \ddagger$ \\
\hline Posterior subcapsular cataract & & 5.5 & & & $7 \cdot 7$ & & 0.08 \\
\hline Daily energy intake (kJ) & 8544.9 & & $2408 \cdot 3$ & $8581 \cdot 3$ & & $2634 \cdot 1$ & 0.79 \\
\hline Dietary fibre $(\mathrm{g} / \mathrm{d})$ & $28 \cdot 3$ & & $10 \cdot 8$ & $28 \cdot 1$ & & 11.6 & 0.81 \\
\hline Vitamin E (mg) & $9 \cdot 5$ & & $7 \cdot 8$ & $8 \cdot 3$ & & $6 \cdot 2$ & $0.001 \ddagger$ \\
\hline Wholegrain consumption (g/d) & $49 \cdot 6$ & & $34 \cdot 8$ & $44 \cdot 2$ & & 34.5 & $0.004 \ddagger$ \\
\hline Legume consumption (g/d) & $18 \cdot 5$ & & 29.9 & $19 \cdot 4$ & & $29 \cdot 3$ & 0.56 \\
\hline
\end{tabular}

* Continuous variables shown as mean values and standard deviations and categorical variables shown as percentages.

$\dagger P$ values based on $t$ tests for means and $\chi^{2}$ for frequencies.

¥ Statistically significant differences $(P<0.05)$.

$\S$ Education defined as trade certificate or higher qualification. 
Table 2. Characteristics according to quintiles of energy-adjusted wholegrain and legume consumption in the Blue Mountains Eye Study (BMES) 2 population* (Mean values and standard deviations; numbers and percentages)

\begin{tabular}{|c|c|c|c|c|c|c|c|c|c|c|c|c|c|c|c|c|}
\hline \multirow[b]{3}{*}{ Characteristics } & \multicolumn{15}{|c|}{ Wholegrain consumption (range g/d) } & \multirow[b]{3}{*}{ P† } \\
\hline & \multicolumn{3}{|c|}{ Quintile $1(<15.7)$} & \multicolumn{3}{|c|}{ Quintile $2(15.7-33.8)$} & \multicolumn{3}{|c|}{ Quintile $3(33.9-50 \cdot 2)$} & \multicolumn{3}{|c|}{ Quintile $4(50 \cdot 3-69 \cdot 9)$} & \multicolumn{3}{|c|}{ Quintile $5(\geq 70.0)$} & \\
\hline & Mean & $\%$ & SD & Mean & $\%$ & SD & Mean & $\%$ & SD & Mean & $\%$ & SD & Mean & $\%$ & SD & \\
\hline$n$ & 287 & & & 295 & & & 323 & & & 329 & & & 307 & & & \\
\hline Age (years) & 67.4 & & $7 \cdot 6$ & 67.5 & & 7.4 & 67.5 & & $7 \cdot 2$ & 68.5 & & 7.3 & 67.9 & & 8.2 & 0.30 \\
\hline Female & & $49 \cdot 1$ & & & $63 \cdot 7$ & & & $61 \cdot 3$ & & & $62 \cdot 6$ & & & 58.6 & & 0.05 \\
\hline Non-smoker & & $42 \cdot 9$ & & & $48 \cdot 3$ & & & $52 \cdot 8$ & & & $55 \cdot 2$ & & & $61 \cdot 6$ & & $<0.001 \ddagger$ \\
\hline Past-smoker & & $42 \cdot 8$ & & & $42 \cdot 8$ & & & $40 \cdot 7$ & & & 38.7 & & & 36.5 & & \\
\hline Current smoker & & $14 \cdot 3$ & & & 8.9 & & & 6.5 & & & $6 \cdot 1$ & & & $2 \cdot 0$ & & \\
\hline Pack/years of smoking & $18 \cdot 8$ & & 24.7 & $13 \cdot 9$ & & $22 \cdot 4$ & $10 \cdot 8$ & & 19.1 & 9.8 & & $18 \cdot 4$ & 7.7 & & $16 \cdot 7$ & $<0.001 \ddagger$ \\
\hline Hypertension & & 54.9 & & & $54 \cdot 1$ & & & $52 \cdot 3$ & & & $49 \cdot 2$ & & & 46.6 & & $0.02 \ddagger$ \\
\hline Diabetes & & $12 \cdot 9$ & & & 9.5 & & & 7.4 & & & $11 \cdot 3$ & & & 9.8 & & 0.45 \\
\hline Education§ & & 53.8 & & & $60 \cdot 1$ & & & 65.7 & & & 64.9 & & & 70.5 & & $<0.001 \neq$ \\
\hline Myopia & & $13 \cdot 2$ & & & 11.9 & & & 14.6 & & & $15 \cdot 2$ & & & $15 \cdot 6$ & & 0.20 \\
\hline Inhaled steroids & & $14 \cdot 0$ & & & $16 \cdot 6$ & & & $15 \cdot 0$ & & & $18 \cdot 3$ & & & 13.4 & & 0.93 \\
\hline BMI (kg/m²) & $27 \cdot 9$ & & 4.7 & $27 \cdot 7$ & & 4.8 & $28 \cdot 0$ & & 4.5 & $27 \cdot 2$ & & 4.7 & 26.8 & & 4.0 & $0.005 \ddagger$ \\
\hline Daily energy intake (kJ) & $9050 \cdot 1$ & & $2770 \cdot 6$ & 8187.8 & & $2567 \cdot 8$ & 8104.5 & & 2191.5 & 8495.9 & & 2085.0 & 8931.6 & & 2290.6 & $<0.001 \ddagger$ \\
\hline \multicolumn{17}{|l|}{ Daily dietary intake $(g) \|$} \\
\hline Fibre & $21 \cdot 6$ & & $6 \cdot 9$ & $25 \cdot 1$ & & $6 \cdot 8$ & $27 \cdot 9$ & & $7 \cdot 0$ & $30 \cdot 3$ & & $8 \cdot 0$ & 33.5 & & $8 \cdot 2$ & $<0.001 \ddagger$ \\
\hline Lutein/zeaxanthin $(\mu \mathrm{g})$ & $775 \cdot 1$ & & $458 \cdot 5$ & 891.8 & & $487 \cdot 2$ & $935 \cdot 1$ & & $462 \cdot 2$ & $997 \cdot 3$ & & 549.6 & 930.4 & & $519 \cdot 0$ & $<0.001 \neq$ \\
\hline Vitamin E (mg) & 6.5 & & $2 \cdot 6$ & $7 \cdot 7$ & & $2 \cdot 8$ & $8 \cdot 1$ & & 4.3 & 8.9 & & $5 \cdot 8$ & $16 \cdot 1$ & & 13.4 & $<0.001 \ddagger$ \\
\hline Vegetables & $416 \cdot 1$ & & 155.4 & $441 \cdot 3$ & & $156 \cdot 6$ & 450.5 & & $145 \cdot 7$ & $467 \cdot 3$ & & $177 \cdot 2$ & 444.0 & & $181 \cdot 0$ & $0.004 \ddagger$ \\
\hline Fruits & 285.5 & & $227 \cdot 7$ & 331.9 & & 224.6 & 369.8 & & $223 \cdot 1$ & 369.6 & & $212 \cdot 1$ & 361.9 & & 199.6 & $<0.001 \ddagger$ \\
\hline Breads/cereals/rice/pasta & $130 \cdot 7$ & & 71.8 & $165 \cdot 3$ & & 87.8 & $212 \cdot 7$ & & 78.9 & $248 \cdot 7$ & & 87.7 & $318 \cdot 2$ & & 112.5 & $<0.001 \ddagger$ \\
\hline Dairy products & $329 \cdot 2$ & & $259 \cdot 3$ & $360 \cdot 6$ & & $214 \cdot 0$ & 377.9 & & $248 \cdot 0$ & $378 \cdot 7$ & & 231.4 & 359.4 & & $226 \cdot 0$ & 0.07 \\
\hline Meats & $152 \cdot 6$ & & 73.7 & $143 \cdot 3$ & & 58.5 & 137.5 & & 58.4 & $130 \cdot 7$ & & $62 \cdot 7$ & $113 \cdot 1$ & & 57.7 & $<0.001 \ddagger$ \\
\hline Eggs & $22 \cdot 4$ & & $20 \cdot 1$ & $22 \cdot 8$ & & 20.4 & 18.7 & & $17 \cdot 0$ & 19.5 & & 17.1 & 15.6 & & 13.7 & $<0.001 \ddagger$ \\
\hline Fish & $26 \cdot 9$ & & $36 \cdot 3$ & 34.1 & & $28 \cdot 8$ & $31 \cdot 2$ & & $27 \cdot 6$ & 34.1 & & $29 \cdot 9$ & $31 \cdot 3$ & & $30 \cdot 8$ & $0.03 \ddagger$ \\
\hline Nuts & 7.4 & & $13 \cdot 8$ & 8.5 & & $12 \cdot 7$ & 7.4 & & 9.7 & $6 \cdot 8$ & & $9 \cdot 3$ & 8.7 & & 11.8 & 0.17 \\
\hline Fats & $17 \cdot 6$ & & $10 \cdot 1$ & $16 \cdot 1$ & & $9 \cdot 2$ & $16 \cdot 2$ & & 9.6 & $15 \cdot 5$ & & $9 \cdot 3$ & $15 \cdot 2$ & & 10.5 & $0.02 \ddagger$ \\
\hline Biscuits/cakes & $45 \cdot 9$ & & 39.4 & $45 \cdot 1$ & & 32.5 & $45 \cdot 2$ & & $34 \cdot 2$ & 44.1 & & $36 \cdot 2$ & 37.6 & & 29.8 & $0.02 \ddagger$ \\
\hline \multirow[t]{4}{*}{ Total diet score } & $8 \cdot 1$ & & $2 \cdot 1$ & $9 \cdot 1$ & & $2 \cdot 0$ & 9.5 & & $2 \cdot 1$ & $10 \cdot 2$ & & 1.9 & $10 \cdot 6$ & & $2 \cdot 1$ & $<0.001 \ddagger$ \\
\hline & \multicolumn{15}{|c|}{ Legume consumption (range $\mathrm{g} / \mathrm{d}$ ) } & \\
\hline & \multicolumn{3}{|c|}{ Quintile $1(<3 \cdot 6)$} & \multicolumn{3}{|c|}{ Quintile $2(3.6-8.6)$} & \multicolumn{3}{|c|}{ Quintile 3 (8.6-13.7) } & \multicolumn{3}{|c|}{ Quintile 4 (13.7-24.6) } & \multicolumn{3}{|c|}{ Quintile 5 (>24.6) } & \\
\hline & Mean & $\%$ & SD & Mean & $\%$ & SD & Mean & $\%$ & SD & Mean & $\%$ & SD & Mean & $\%$ & SD & $P+$ \\
\hline$n$ & 303 & & & 312 & & & 322 & & & 303 & & & 301 & & & \\
\hline Age (years) & 69.0 & & $7 \cdot 7$ & 68.7 & & 7.3 & $67 \cdot 2$ & & 7.9 & 66.5 & & $7 \cdot 3$ & 67.5 & & $7 \cdot 3$ & $<0.001 \ddagger$ \\
\hline Female & & $51 \cdot 2$ & & & $60 \cdot 6$ & & & $61 \cdot 2$ & & & $62 \cdot 1$ & & & $61 \cdot 1$ & & $0.02 \ddagger$ \\
\hline Non-smoker & & 53.8 & & & 53.53 & & & 50.9 & & & 53.5 & & & $50 \cdot 0$ & & 0.46 \\
\hline Past-smoker & & $40 \cdot 3$ & & & 39.1 & & & 39.1 & & & 39.2 & & & $43 \cdot 7$ & & \\
\hline Current smoker & & 5.9 & & & 7.4 & & & $10 \cdot 0$ & & & 7.3 & & & $6 \cdot 3$ & & \\
\hline Pack/years of smoking & 11.0 & & $20 \cdot 6$ & 11.9 & & $20 \cdot 4$ & $12 \cdot 2$ & & $20 \cdot 1$ & $12 \cdot 5$ & & $20 \cdot 8$ & $12 \cdot 7$ & & 21.6 & 0.86 \\
\hline
\end{tabular}




\section{NS British Journal of Nutrition}

Table 2. (Continued)

\begin{tabular}{|c|c|c|c|c|c|c|c|c|c|c|c|c|c|c|c|c|}
\hline & \multicolumn{15}{|c|}{ Legume consumption (range g/d) } & \multirow[b]{3}{*}{$P \dagger$} \\
\hline & \multicolumn{3}{|c|}{ Quintile $1(<3.6)$} & \multicolumn{3}{|c|}{ Quintile $2(3.6-8.6)$} & \multicolumn{3}{|c|}{ Quintile $3(8.6-13.7)$} & \multicolumn{3}{|c|}{ Quintile 4 (13.7-24.6) } & \multicolumn{3}{|c|}{ Quintile 5 (>24.6) } & \\
\hline & Mean & $\%$ & SD & Mean & $\%$ & SD & Mean & $\%$ & SD & Mean & $\%$ & SD & Mean & $\%$ & SD & \\
\hline Hypertension & & $45 \cdot c$ & & & 53.2 & & & 52.5 & & & $49 \cdot 3$ & & & 56.5 & & $0.04 \ddagger$ \\
\hline Diabetes & & $9 \cdot 2$ & & & $10 \cdot 0$ & & & 11.5 & & & $8 \cdot 6$ & & & $11 \cdot 3$ & & 0.62 \\
\hline Education§ & & $65 \cdot \varepsilon$ & & & 61.2 & & & $65 \cdot 0$ & & & $60 \cdot 2$ & & & $63 \cdot 7$ & & 0.56 \\
\hline Myopia & & 12.9 & & & $15 \cdot 1$ & & & 16.5 & & & 11.9 & & & $14 \cdot 3$ & & 0.96 \\
\hline Inhaled steroids & & 14.3 & & & $16 \cdot 3$ & & & 13.8 & & & 17.5 & & & $15 \cdot 8$ & & 0.54 \\
\hline BMl $\left(\mathrm{kg} / \mathrm{m}^{2}\right)$ & $27 \cdot 1$ & & 3.8 & $27 \cdot 5$ & & 4.6 & $28 \cdot 1$ & & 4.6 & $27 \cdot 4$ & & 4.8 & 27.5 & & 4.8 & 0.11 \\
\hline Daily energy intake (kJ) & $10296 \cdot 7$ & & 2063.6 & $8078 \cdot 2$ & & $2069 \cdot 4$ & 8011.6 & & 2226.4 & 7723.9 & & 1891.4 & $8662 \cdot 0$ & & $2795 \cdot 7$ & $<0.001 \ddagger$ \\
\hline \multicolumn{17}{|l|}{ Daily dietary intake $(\mathrm{g}) \|$} \\
\hline Fibre & $26 \cdot 1$ & & 9.5 & $26 \cdot 3$ & & 6.7 & $26 \cdot 6$ & & $7 \cdot 2$ & $28 \cdot 0$ & & 7.5 & 32.4 & & 9.5 & $<0.001 \ddagger$ \\
\hline Lutein/zeaxanthin $(\mu \mathrm{g})$ & $826 \cdot 3$ & & 503.4 & 871.4 & & 433.7 & 894.9 & & 449.9 & 873.7 & & $425 \cdot 1$ & 1083.7 & & $636 \cdot 7$ & $<0.001 \ddagger$ \\
\hline Vitamin E (mg) & $10 \cdot 3$ & & $10 \cdot 1$ & $9 \cdot 1$ & & $6 \cdot 7$ & $9 \cdot 1$ & & 6.5 & 9.5 & & $7 \cdot 3$ & 9.4 & & 7.9 & 0.28 \\
\hline Vegetables & 401.9 & & 161.6 & 426.8 & & $148 \cdot 6$ & 421.4 & & $135 \cdot 6$ & $440 \cdot 3$ & & $139 \cdot 8$ & 535.5 & & 198.4 & $<0.001 \ddagger$ \\
\hline Fruits & 318.4 & & 235.0 & 328.8 & & 214.7 & 330.5 & & $200 \cdot 6$ & 347.6 & & $198 \cdot 3$ & $402 \cdot 7$ & & 238.2 & $<0.001 \ddagger$ \\
\hline Breads/cereals/rice/pasta & $196 \cdot 6$ & & $111 \cdot 1$ & 211.0 & & 97.8 & $212 \cdot 6$ & & 95.8 & $223 \cdot 1$ & & $105 \cdot 0$ & $242 \cdot 6$ & & $131 \cdot 8$ & $<0.001 \ddagger$ \\
\hline Dairy products & $363 \cdot 1$ & & 269.8 & $361 \cdot 2$ & & 231.3 & 368.5 & & 232.4 & 352.6 & & 211.5 & 364.3 & & 235.6 & 0.95 \\
\hline Meats & 140.9 & & 72.5 & 141.8 & & 59.4 & 139.1 & & $59 \cdot 4$ & $133 \cdot 3$ & & 54.2 & $120 \cdot 0$ & & 67.0 & $<0.001 \ddagger$ \\
\hline Eggs & $17 \cdot 8$ & & $19 \cdot 2$ & $18 \cdot 4$ & & $16 \cdot 0$ & $21 \cdot 2$ & & $18 \cdot 8$ & $20 \cdot 3$ & & $16 \cdot 8$ & 20.9 & & 18.5 & 0.06 \\
\hline Fish & $25 \cdot 1$ & & 31.3 & $26 \cdot 8$ & & 21.8 & 29.8 & & 24.7 & 33.5 & & 28.4 & 43.0 & & 41.4 & $<0.001 \ddagger$ \\
\hline Nuts & $7 \cdot 4$ & & 14.1 & 7.2 & & 10.3 & 8.1 & & 11.9 & 7.4 & & $7 \cdot 7$ & 8.6 & & 12.5 & 0.53 \\
\hline Fats & $17 \cdot 3$ & & $10 \cdot 0$ & 17.5 & & 9.6 & $16 \cdot 1$ & & $10 \cdot 0$ & $15 \cdot 7$ & & $9 \cdot 3$ & $13 \cdot 8$ & & 9.4 & $<0.001 \ddagger$ \\
\hline Biscuits/cakes & $45 \cdot 6$ & & $45 \cdot 4$ & $42 \cdot 6$ & & $25 \cdot 8$ & $46 \cdot 4$ & & 33.9 & $44 \cdot 8$ & & $29 \cdot 3$ & 38.2 & & 35.5 & $0.03 \ddagger$ \\
\hline Total diet score & 9.0 & & $2 \cdot 1$ & 9.2 & & $2 \cdot 0$ & $9 \cdot 4$ & & $2 \cdot 1$ & 9.7 & & $2 \cdot 2$ & $10 \cdot 4$ & & $2 \cdot 2 \ddagger$ & $<0.001 \ddagger$ \\
\hline
\end{tabular}


(Table 2). Overall, wholegrain and legume consumptions were positively associated with consumptions of majority of healthy foods and high adherence to dietary guidelines (total diet score) in this population, with significant $P$ values for trend across quintiles of wholegrain and legume consumptions (Table 2). However, correlations between consumptions of whole grains or legumes and these food groups were low $(r<0.3)$, except for the correlation between consumptions of whole grains and breads/cereals/rice/pasta ( $r$ 0.6). Baseline characteristics by quintiles of energy-adjusted wholegrain and legume consumption of BMES 2 participants at-risk of different types of cataract did not differ much from the entire population (online Supplementary Table S1).

After excluding those with cataract at the BMES 2 examination, there were 852, 522 and 1011 participants at risk of incident cortical, nuclear and PSC cataract, respectively. The 5-year incidence of cortical, nuclear and PSC cataract was 18.2, 16.5 and $5.9 \%$, respectively.

After multivariable adjustment, there were no significant associations between total wholegrain consumption and any type of incident cataract (Table 3). Further analysis using different sources of whole grain also showed no significant associations between consumption of any main source of whole grain and incidence of any type of cataract (Table 4).

The associations between legume consumption and incidence of the three cataract types are shown in Table 5. A protective association between the highest quintile consumption of legumes and incident PSC cataract was observed, compared with the lowest quintile (adjusted OR for Q5 $v$. Q1, 0.37, $95 \%$ CI 0·15, 0.92) after adjustment for age, sex, smoking status, education, inhaled steroid use and myopia. However, there was no significant trend of this association across the quintiles $(P=0.08)$ (Table 5). No associations were observed between legume consumption and incidence of cortical or nuclear cataract (Table 5).

Findings were essentially the same when further adjustment for daily energy intake (as a continuous variable or as quintiles) in the logistic models in a supplementary analysis (data not shown)

\section{Discussion}

In this population-based sample of older Australians, we found no association between wholegrain consumption at baseline and 5-year incidence of any of the three types of cataract. However, a protective association for incident PSC cataract was found among those in the highest quintile of legume consumption compared with those in the lowest quintile.

Whole grains are slow-release carbohydrates with strong antioxidant activity and a good source of vitamins and minerals ${ }^{(10,29)}$. Wholegrain consumption has been linked with reduced risk of many chronic diseases such as $\mathrm{CHD}$, stroke and diabetes $^{(12-14)}$. Age-related cataract also involves chronic processes of progressive degeneration of lens proteins, closely linked with ageing process, resulting in loss of lens transparency ${ }^{(1)}$. Moreover, all these chronic diseases, including age-related cataract, have been linked to oxidative processes and inflammation that accumulate over time ${ }^{(30,31)}$. Given the high nutritional content and antioxidant property of whole grains ${ }^{(10,29)}$, and that cataractogenesis involves oxidation processes ${ }^{(32,33)}$, we speculated that high consumption of whole grain in daily diet may benefit overall health including the eye and ageing process.

Previous cross-sectional and longitudinal studies that investigated the association between whole grains and cataract were mainly conducted in women. In the Harvard Nurses' Health Study, those who had high consumption of whole grains were less likely to have nuclear opacities than those with low consumption, although the difference was not statistically significant ${ }^{(34)}$. Low diet quality, including low intake of grains, was related to higher prevalence of nuclear cataract in an ancillary study of the Women's Health Initiative ${ }^{(35)}$. Whole grains were a main contributor (17\%) to the total antioxidant capacity in diet, and increasing total antioxidant capacity was found associated with decreased risk of incident cataract in a cohort of women $^{(36)}$. There has been no other study reporting on the longitudinal associations between whole grains and age-related cataract in other general populations. In the present study of our BMES cohort, we did not observe longitudinal associations

Table 3. Association between energy-adjusted total wholegrain consumption and 5-year incidence of cortical, nuclear and posterior subcapsular (PSC) cataract in the Blue Mountains Eye Study (BMES)

(Numbers and percentages; odds ratios and $95 \%$ confidence intervals)

\begin{tabular}{|c|c|c|c|c|c|c|c|c|c|c|c|c|}
\hline \multirow[b]{2}{*}{$\begin{array}{l}\text { Wholegrain } \\
\text { consumption }\end{array}$} & \multicolumn{4}{|c|}{ Cortical cataract } & \multicolumn{4}{|c|}{ Nuclear cataract } & \multicolumn{4}{|c|}{ PSC cataract } \\
\hline & $n / N$ & $\%$ & $\begin{array}{l}\text { Adjusted } \\
\mathrm{OR}^{*}\end{array}$ & $95 \% \mathrm{Cl}$ & $n / N$ & $\%$ & $\begin{array}{c}\text { Adjusted } \\
\text { OR† }\end{array}$ & $95 \% \mathrm{Cl}$ & $n / N$ & $\%$ & $\begin{array}{l}\text { Adjusted } \\
\text { OR } \ddagger\end{array}$ & $95 \% \mathrm{Cl}$ \\
\hline Quintile 1 & $30 / 167$ & $18 \cdot 0$ & 1.0 (ref) & & $15 / 96$ & $15 \cdot 6$ & 1.0 (ref) & & $11 / 197$ & $5 \cdot 6$ & 1.0 (ref) & \\
\hline Quintile 2 & 29/152 & $19 \cdot 1$ & 1.05 & $0.59,1.87$ & $17 / 96$ & $17 \cdot 7$ & 1.08 & $0.47,2.48$ & $5 / 191$ & $2 \cdot 6$ & 0.44 & $0 \cdot 15,1 \cdot 33$ \\
\hline Quintile 3 & $26 / 177$ & $14 \cdot 7$ & 0.72 & $0.40,1.29$ & $17 / 113$ & $15 \cdot 0$ & 0.90 & $0.40,2.05$ & $22 / 210$ & 10.5 & 1.64 & $0.75,3.61$ \\
\hline Quintile 4 & $40 / 184$ & $21 \cdot 7$ & $1 \cdot 19$ & $0.68,2.01$ & $27 / 118$ & $22 \cdot 9$ & 1.24 & $0.56,2.75$ & $7 / 213$ & $3 \cdot 3$ & 0.43 & $0.16,1.17$ \\
\hline Quintile 5 & $30 / 172$ & 17.4 & 0.90 & $0.51,1.60$ & $10 / 99$ & $10 \cdot 1$ & 0.82 & $0.32,2 \cdot 14$ & $15 / 200$ & 7.5 & 0.97 & $0.42,2.26$ \\
\hline$P_{\text {for trend }}$ & & & 0.90 & & & & 0.88 & & & & 0.92 & \\
\hline $\begin{array}{l}\text { Per } 8 \mathrm{~g} \text { serve/d } \\
\text { increase§ }\end{array}$ & $155 / 852$ & $18 \cdot 2$ & 0.99 & $0.95,1.04$ & $86 / 522$ & $16 \cdot 5$ & 1.00 & $0.93,1.08$ & $60 / 1011$ & $5 \cdot 9$ & 1.01 & $0.96,1.08$ \\
\hline
\end{tabular}

$n / N$, number of cases/number at risk.

* Parsimonious model for cortical cataract adjusted for age, sex and smoking status (past, current and never smokers) at BMES 2.

† Parsimonious model for nuclear cataract adjusted for age, sex, smoking status and energy-adjusted vitamin E intake at BMES 2.

$\ddagger$ Parsimonious model for PSC cataract adjusted for age, sex, smoking status, education, inhaled steroid use and myopia at BMES 2.

$\S$ Weight per serve estimated from wholegrain component within foods consumed. 
Table 4. Association between energy-adjusted wholegrain consumption from different sources and 5-year incidence of cortical, nuclear and posterior subcapsular (PSC) cataract in the Blue Mountains Eye Study (BMES)

(Odds ratios and $95 \%$ confidence intervals)

\begin{tabular}{|c|c|c|c|c|c|c|c|c|c|}
\hline \multirow[b]{2}{*}{ Wholegrain consumption } & \multicolumn{3}{|c|}{ Cortical cataract } & \multicolumn{3}{|c|}{ Nuclear cataract } & \multicolumn{3}{|c|}{ PSC cataract } \\
\hline & Adjusted OR* & $95 \% \mathrm{Cl}$ & $P_{\text {for trend }}$ & Adjusted OR $†$ & $95 \% \mathrm{Cl}$ & $P_{\text {for trend }}$ & Adjusted OR $\ddagger$ & $95 \% \mathrm{Cl}$ & $P_{\text {for trend }}$ \\
\hline \multicolumn{10}{|l|}{ Oatmeal } \\
\hline Quintile 1 & $1.0(\mathrm{ref})$ & & 0.21 & $1.0(\mathrm{ref})$ & & 0.81 & 1.0 (ref) & & 0.65 \\
\hline Quintile 2 & $1 \cdot 15$ & $0.66,1.99$ & & $1 \cdot 13$ & $0.52,2.46$ & & 1.33 & $0.59,3.00$ & \\
\hline Quintile 3 & 0.87 & $0.49,1.54$ & & 0.70 & $0.31,1.61$ & & 0.97 & $0.41,2.26$ & \\
\hline Quintile 4 & 1.07 & $0.62,1.84$ & & 0.79 & $0.36,1.72$ & & 0.54 & $0.21,1.41$ & \\
\hline Quintile 5 & 0.71 & $0.39,1 \cdot 28$ & & 0.90 & $0.41,1.98$ & & 1.00 & $0.44,2 \cdot 30$ & \\
\hline Per $8 \mathrm{~g}$ serve/d increase§ & 0.96 & $0.88,1.05$ & - & 0.99 & $0.87,1.12$ & - & 1.00 & $0.81,1.26$ & - \\
\hline \multicolumn{10}{|l|}{ Wholemeal/multigrain bread } \\
\hline Quintile 1 & 1.0 (ref) & & 0.55 & 1.0 (ref) & & 0.45 & 1.0 (ref) & & 0.38 \\
\hline Quintile 2 & 1.26 & $0.72,2 \cdot 21$ & & 1.47 & $0.62,3.52$ & & 1.08 & $0.43,2.74$ & \\
\hline Quintile 3 & 1.08 & $0.60,1.94$ & & 1.88 & $0.78,4.50$ & & 0.84 & $0.32,2 \cdot 24$ & \\
\hline Quintile 4 & 1.03 & $0.58,1.81$ & & 1.67 & $0.73,3.82$ & & 1.21 & $0.50,2.91$ & \\
\hline Quintile 5 & 0.96 & $0.54,1.70$ & & 1.57 & $0.68,3.61$ & & 1.33 & $0.55,3.18$ & \\
\hline Per 8 g serve/d increase & 0.97 & $0.88,1.05$ & - & 1.06 & $0.94,1.19$ & - & 1.06 & $0.93,1.20$ & - \\
\hline \multicolumn{10}{|l|}{ Breakfast cereal } \\
\hline Quintile 1 & 1.0 (ref) & & 0.24 & 1.0 (ref) & & 0.21 & 1.0 (ref) & & 0.75 \\
\hline Quintile 2 & 1.30 & $0.65,2.58$ & & 0.83 & $0.35,1.94$ & & 0.54 & $0.18,1.67$ & \\
\hline Quintile 3 & 1.34 & $0.68,2.63$ & & 0.90 & $0.39,2.09$ & & 1.34 & $0.55,3.26$ & \\
\hline Quintile 4 & $1 \cdot 15$ & $0.58,2 \cdot 30$ & & 0.55 & $0.22,1.36$ & & 0.61 & $0.22,1.68$ & \\
\hline Quintile 5 & 1.62 & $0.83,3.14$ & & 0.57 & $0.18,1.82$ & & 0.85 & $0.33,2.17$ & \\
\hline Per $8 \mathrm{~g}$ serve/d increase§ & 1.03 & $0.97,1.10$ & - & 0.88 & $0.75,1.04$ & - & 0.93 & $0.83,1.04$ & - \\
\hline \multicolumn{10}{|l|}{ Brown rice } \\
\hline Quintile 1 & $1.0(\mathrm{ref})$ & & 0.46 & 1.0 (ref) & & 0.29 & 1.0 (ref) & & 0.55 \\
\hline Quintile 2 & 1.07 & $0.59,1.95$ & & 1.45 & $0.66,3.20$ & & 1.60 & $0.70,3.67$ & \\
\hline Quintile 3 & $1 \cdot 14$ & $0.62,2.08$ & & 1.00 & $0.41,2.41$ & & 0.95 & $0.38,2.37$ & \\
\hline Quintile 4 & 1.61 & $0.91,2.84$ & & 0.69 & $0.28,1.68$ & & 0.63 & $0.24,1.64$ & \\
\hline Quintile 5 & 0.95 & $0.52,1.71$ & & 1.53 & $0.68,3.48$ & & 0.89 & $0.38,2 \cdot 10$ & \\
\hline Per $8 \mathrm{~g}$ serve/d increase $\S$ & 0.91 & $0.72,1.13$ & - & $1 \cdot 10$ & $0.92,1.31$ & - & $1 \cdot 10$ & $0.95,1.26$ & - \\
\hline
\end{tabular}

* Parsimonious model for cortical cataract adjusted for age, sex and smoking status at BMES 2.

† Parsimonious model for nuclear cataract adjusted for age, sex, smoking status and energy-adjusted vitamin E intake at BMES 2.

$\ddagger$ Parsimonious model for PSC cataract adjusted for age, sex, smoking status, education, inhaled steroid use and myopia at BMES 2.

$\S$ Weight per serve estimated from wholegrain component within foods consumed.

Table 5. Association between energy-adjusted legume consumption and 5-year incidence of cortical, nuclear and posterior subcapsular (PSC) cataract in the Blue Mountains Eye Study (BMES)

(Numbers and percentages; odds ratios and $95 \%$ confidence intervals)

\begin{tabular}{|c|c|c|c|c|c|c|c|c|c|c|c|c|}
\hline \multirow[b]{2}{*}{ Legume consumption } & \multicolumn{4}{|c|}{ Cortical cataract } & \multicolumn{4}{|c|}{ Nuclear cataract } & \multicolumn{4}{|c|}{ PSC cataract } \\
\hline & $n / N$ & $\%$ & $\begin{array}{l}\text { Adjusted } \\
\text { OR }^{*}\end{array}$ & $95 \% \mathrm{Cl}$ & $n / N$ & $\%$ & $\begin{array}{l}\text { Adjusted } \\
\text { OR† }\end{array}$ & $95 \% \mathrm{Cl}$ & $n / N$ & $\%$ & $\begin{array}{l}\text { Adjusted } \\
\text { OR } \ddagger\end{array}$ & $95 \% \mathrm{Cl}$ \\
\hline Quintile 1 & $32 / 166$ & $19 \cdot 3$ & 1.0 (ref) & & $17 / 88$ & $19 \cdot 3$ & 1.0 (ref) & & $18 / 196$ & $9 \cdot 2$ & 1.0 (ref) & \\
\hline Quintile 2 & $29 / 175$ & $16 \cdot 6$ & 0.80 & $0.45,1.40$ & $15 / 82$ & $18 \cdot 3$ & 0.83 & $0.36,1.90$ & $10 / 202$ & $5 \cdot 0$ & 0.52 & $0.23,1.18$ \\
\hline Quintile 3 & $36 / 178$ & $20 \cdot 2$ & $1 \cdot 10$ & $0.64,1.89$ & $15 / 128$ & 11.7 & 0.55 & $0.25,1.24$ & $12 / 211$ & $5 \cdot 7$ & 0.62 & $0.28,1.35$ \\
\hline Quintile 4 & $30 / 176$ & $17 \cdot 1$ & 0.90 & $0.51,1.58$ & $17 / 111$ & $15 \cdot 3$ & 0.80 & $0.36,1.79$ & $13 / 204$ & $6 \cdot 4$ & 0.71 & $0.32,1.56$ \\
\hline Quintile 5 & $28 / 157$ & $17 \cdot 8$ & 0.97 & $0.55,1.72$ & $22 / 113$ & 19.5 & 1.04 & $0.48,2.24$ & $7 / 198$ & 3.5 & $0.37 \S$ & $0.15,0.92$ \\
\hline$P_{\text {for trend }}$ & & & 0.93 & & & & 0.44 & & & & 0.08 & \\
\hline $\begin{array}{l}\text { Per } 75 \mathrm{~g} \text { serve/d } \\
\text { increasell }\end{array}$ & $155 / 852$ & $18 \cdot 2$ & 1.32 & $0.84,2.07$ & $86 / 522$ & 16.5 & 1.13 & $0.60,2 \cdot 15$ & $60 / 1011$ & 5.9 & 1.02 & $0.59,1.75$ \\
\hline
\end{tabular}

$n / N$, number of cases/number at risk.

* Parsimonious model for cortical cataract adjusted for age, sex and smoking status at BMES 2.

† Parsimonious model for nuclear cataract adjusted for age, sex, smoking status, and energy-adjusted vitamin E intake at BMES 2.

‡ Parsimonious model for PSC cataract adjusted for age, sex, smoking status, education, inhaled steroid use and myopia at BMES 2.

$\S$ Statistical significance $(P<0.05)$.

II Weight per serve estimated from legume component within foods consumed.

between wholegrain consumption at baseline and the 5-year risk of developing any type of age-related cataract.

Besides the possibility of no association, the lack of longitudinal association in our study sample could also be due to the overall low consumption level of whole grain among the BMES participants, with $<50 \%$ of the population reaching the daily target intake of $48 \mathrm{~g}$. A recent report using 2011-2013 Australian Health Survey data indicated only $42.4 \%$ of older 
Australians aged $\geq 51$ years reached the recommended wholegrain daily intake target ${ }^{(37)}$. Recent reports from the US and the UK also indicated low level of wholegrain consumption in these countries among all age groups, including older adults $^{(38,39)}$

Previous research has suggested that individual wholegrain foods may have differing effects, due to differing nutrient composition $^{(40,41)}$ and $\mathrm{GI}^{(42)}$ of individual wholegrain foods. For example, antioxidant activity is higher in wheat $(76.7 \mu \mathrm{mol} / \mathrm{g}$ of grain) and oats $(74.7 \mu \mathrm{mol} / \mathrm{g}$ of grain) compared with brown rice $(55.8 \mu \mathrm{mol} / \mathrm{g} \text { of grain })^{(43)}$, while average GI was higher in whole wheat bread $(\mathrm{GI}=74)$ and brown rice $(\mathrm{GI}=68)$ than rolled oats $(\mathrm{GI}=55)^{(9)}$. It was speculated that different sources of whole grains may affect different types of cataract: for example, lower GI of rolled oats may be protectively associated with cortical or PSC cataract, which are most linked with diabetes and glucose levels ${ }^{(1)}$; whereas wholegrain sources with high antioxidant activity may be protectively associated with nuclear cataract ${ }^{(33)}$. However, in this older Australian population, we did not observe any association of cataract incidence with different food sources of whole grain. Again, the overall low consumption of whole grains in this population could have limited our ability to assess individual wholegrain sources.

High consumption of legumes has been linked to lower risk of diabetes ${ }^{(16)}, \mathrm{CVD}^{(10,15)}$ and mortality ${ }^{(17)}$. We found that BMES participants in the highest quintile of legume intake, compared with those in the lowest, appeared to have lower incidence of PSC cataract. Current dietary guidelines do not include recommended daily legume intake amount but suggest that legumes contribute to overall vegetable consumption as part of a healthy diet and the Grains \& Legumes Nutrition Council recommends weekly consumption of $2-3$ times $^{(28)}$ with one serve of legumes equal to $75 \mathrm{~g}$ (1/2 cup) of cooked, dried or canned beans, chickpeas or lentils. The minimum intake for legumes in the highest quintile in our study population is $24.6 \mathrm{~g} / \mathrm{d}$ ( $172.2 \mathrm{~g} /$ week) equivalent to roughly 2.3 serves/week, meeting the recommended intake level. While we cannot exclude the possibility of chance finding for this association, our observation is in keeping with findings from a previous study in India showing high prevalence of senile cataract and cataract surgery associated with low intake of beans and lentils ${ }^{(44)}$.

In the same BMES population, we have previously shown a protective association between high intakes of protein and incident PSC cataract ${ }^{(45)}$. The India-US case-control study also found increasing intake of protein associated with decreased prevalence of PSC $^{(46)}$. Legumes are a good source of protein and amino acids. In particular, legumes are rich in lysine, an essential amino $\operatorname{acid}^{(47)}$. A previous study showed that high levels of lysine and other amino acids in the blood were associated with decreased odds of PSC in a case-control study ${ }^{(48)}$. Although animal studies documented beneficial effect of lysine on cataract formation ${ }^{(49,50)}$, and findings from previous clinic-based studies showed promising effect of Bendazac lysine on cataract ${ }^{(51,52)}$, such findings appeared not to have been translated into preventive or therapeutic management of cataract.

Legumes are also a good source of essential micronutrients, such as thiamin, niacin, folate, riboflavin, Zn, vitamins $\mathrm{E}$ and A, and fibre ${ }^{(47,53)}$. Previous epidemiological studies investigating micronutrient associations with cataract reported mixed findings ${ }^{(4-7,54)}$. Riboflavin has been shown to have a protective association with nuclear ${ }^{(4,6)}$ and $\operatorname{cortical}^{(5,54)}$ but not PSC cataract. We could not find evidence of associations between essential micronutrients and PSC cataract from the literature. A previous BMES report documented no associations between dietary fibre intake and any incident cataract type ${ }^{(45)}$, while the Beaver Dam Eye Study reported decreased prevalence of nuclear cataract among men with high intakes of fibre ${ }^{(6)}$. In the present study, further adjustment with dietary fibre did not alter the association between PSC and legume intake (data not shown).

A limitation for our study is that we used only four food items to estimate wholegrain consumption. We could have underestimated wholegrain consumption in the study population as we did not include wholegrain content of discretionary snack foods such as popcorn or savoury biscuits, although these snack foods are not consumed daily. The Australian wholegrain database $^{(55,56)}$ is comprehensive in terms of the foods that have been included. However, during the late 1990s when the BMES 2 participants were examined, wholegrain food options were limited, with much less options available as it is now. We compared the BMES FFQ dataset against the Australian wholegrain database ${ }^{(55,56)}$ and found that most wholegrain percentages were similar between the two datasets. However, there were inconsistencies between BMES FFQ dataset and the Australian wholegrain database in regard to the serving sizes of some breakfast cereals. As the serving sizes used in BMES were from the NUTTAB95 ${ }^{(22)}$, no further amendments were made to the BMES wholegrain estimates. Nevertheless, we feel that we have covered the major sources of whole grains available in the $1990 \mathrm{~s}$ and estimated the consumption level as accurately as possible for the BMES population. We cannot exclude potential confounding from unknown or unmeasured confounders such as UV or sunlight exposure.

Strengths of our study include its longitudinal study design, the standardised methods used during each examination, masked grading of cataract using lens photographs with high reproducibility ${ }^{(20)}$ and good follow-up of participants $(87.4 \%$ of survivors were re-examined).

In summary, we found no significant associations of total wholegrain intake or consumption of wholegrain-rich food items at baseline with the 5-year incidence of any of the three cataract types in this older Australian population. We found high consumption level of legumes associated with decreased incidence of PSC, and replication of this association in other studies is needed.

\section{Acknowledgements}

This work was supported by the Australian National Health and Medical Research Council (grant numbers 974159, 211069 and 1031058) (A. G. T., grant number GNT1094094). The Australian National Health and Medical Research Council had no role in the design, analysis or writing of this article.

A. G. T., J. J. W. and V. M. F. formulated the research question; R. G. C. and P. M. designed the BMES cohort study; A. G. T., J. J. 
W., J. R. and V. M. F. conducted this particular research; A. G. T. and A. K. analysed data or performed statistical analyses; A. G. T., V. M. F. and J. J. W. wrote and edited the manuscript; A. G. T. and J. J. W. had primary responsibility for final content. All authors revised the text critically for important intellectual content, contributed to and approved the final manuscript.

The authors declare that there are no conflicts of interest.

\section{Supplementary material}

For supplementary material referred to in this article, please visit https://doi.org/10.1017/S000711452000104X

\section{References}

1. Asbell PA, Dualan I, Mindel J, et al. (2005) Age-related cataract. Lancet 365, 599-609.

2. Liu Y-C, Wilkins M, Kim T, et al. (2017) Cataracts. Lancet 390 , 600-612.

3. Flaxman SR, Bourne RRA, Resnikoff S, et al. (2017) Global causes of blindness and distance vision impairment 1990-2020: a systematic review and meta-analysis. Lancet Glob Health $\mathbf{5}$, e1221-e1234.

4. Jacques PF, Chylack LT Jr, Hankinson SE, et al. (2001) Long-term nutrient intake and early age-related nuclear lens opacities. Arch Ophthalmol 119, 1009-1019.

5. Leske MC, Chylack LT \& Wu SY (1991) The lens opacities case-control study. Risk factors for cataract. Arch Ophthalmol 109, 244-251.

6. Mares-Perlman JA, Brady WE, Klein BE, et al. (1995) Diet and nuclear lens opacities. Am J Epidemiol 141, 322-334.

7. Tan AG, Mitchell P, Flood VM, et al. (2008) Antioxidant nutrient intake and the long-term incidence of age-related cataract: the Blue Mountains Eye Study. Am J Clin Nutr 87, 1899-1905.

8. Chew EY (2013) Nutrition effects on ocular diseases in the aging eye. Invest Ophthalmol Vis Sci 54, ORSF42-ORSF47.

9. Atkinson FS, Foster-Powell K \& Brand-Miller JC (2008) International tables of glycemic index and glycemic load values: 2008. Diabetes Care 31, 2281-2283.

10. Flight I \& Clifton P (2006) Cereal grains and legumes in the prevention of coronary heart disease and stroke: a review of the literature. Eur J Clin Nutr 60, 1145-1159.

11. Djordjevic TM, Šiler-Marinkovic SS \& Dimitrijevic-Brankovic SI (2011) Antioxidant activity and total phenolic content in some cereals and legumes. Int J Food Prop 14, 175-184.

12. Ye EQ, Chacko SA, Chou EL, et al. (2012) Greater wholegrain intake is associated with lower risk of type 2 diabetes, cardiovascular disease, and weight gain. J Nutr 142, 1304-1313.

13. Aune D, Keum N, Giovannucci E, et al. (2016) Whole grain consumption and risk of cardiovascular disease, cancer, and all cause and cause specific mortality: systematic review and dose-response meta-analysis of prospective studies. BMJ 353, i2716.

14. Harland JI \& Garton LE (2008) Wholegrain intake as a marker of healthy body weight and adiposity. Public Health Nutr 11 , 554-563.

15. Marventano S, Izquierdo Pulido M, Sanchez-Gonzalez C, et al. (2017) Legume consumption and CVD risk: a systematic review and meta-analysis. Public Health Nutr 20, 245-254.

16. Becerra-Tomas N, Diaz-Lopez A, Rosique-Esteban $\mathrm{N}$, et al. (2018) Legume consumption is inversely associated with type 2 diabetes incidence in adults: a prospective assessment from the PREDIMED study. Clin Nutr 37, 906-913.
17. Papandreou C, Becerra-Tomas N, Bullo M, et al. (2018) Legume consumption and risk of all-cause, cardiovascular, and cancer mortality in the PREDIMED study. Clin Nutr 38, 348-356.

18. Mitchell P, Cumming RG, Attebo K, et al. (1997) Prevalence of cataract in Australia. Ophthalmology 104, 581-588.

19. Klein BE, Klein R, Linton KL, et al. (1990) Assessment of cataracts from photographs in the Beaver Dam Eye Study. Ophthalmology 97, 1428-1433.

20. Kanthan GL, Wang JJ, Rochtchina E, et al. (2008) Ten-year incidence of age-related cataract and cataract surgery in an older Australian population. The Blue Mountains Eye Study. Ophthalmology 115, 808-814.

21. Smith W, Mitchell P, Reay EM, et al. (1998) Validity and reproducibility of a self-administered food frequency questionnaire in older people. Aust N Z J Public Health 22, 456-463.

22. Department of Community Services and Health (1995) NUTTAB95 Nutrient Data Table for Use in Australia. Canberra: Australian Government Publishing Service.

23. Go Grains Health \& Nutrition (2010) The Grains \& Legumes Health Report. Spit Junction, NSW: Go Grains Health \& Nutrition Ltd.

24. Willett W \& Stampfer MJ (1986) Total energy intake: implications for epidemiologic analyses. Am J Epidemiol 124, 17-27.

25. Russell J, Flood V, Rochtchina E, et al. (2013) Adherence to dietary guidelines and 15-year risk of all-cause mortality. $\mathrm{Br} \mathrm{J}$ Nutr 109, 547-555.

26. Grains \& Legumes Nutrition Council (2018) Recommended amount of grains. https://www.glnc.org.au/grains-2/ recommended-amounts/recommended-amount-of-grains/ (accessed January 2020)

27. National Health and Medical Research Council (2013) Australian Dietary Guidelines. Canberra: National Health and Medical Research Council.

28. Grains \& Legumes Nutrition Council (2018) Recommended amounts of legumes. https://www.glnc.org.au/grains-2/ recommended-amounts/recommended-amount-of-legumes/ (accessed July 2018)

29. Slavin JL, Martini MC, Jacobs DR Jr, et al. (1999) Plausible mechanisms for the protectiveness of whole grains. $A m J$ Clin Nutr 70, 459S-463S.

30. Franceschi $\mathrm{C}$, Garagnani $\mathrm{P}$, Parini $\mathrm{P}$, et al. (2018) Inflammaging: a new immune-metabolic viewpoint for age-related diseases. Nat Rev Endocrinol 14, 576-590.

31. Liguori I, Russo G, Curcio F, et al. (2018) Oxidative stress, aging, and diseases. Clin Interv Aging 13, 757-772.

32. Beebe DC, Holekamp NM \& Shui YB (2010) Oxidative damage and the prevention of age-related cataracts. Ophthalmic Res $\mathbf{4 4}$, $155-165$

33. Truscott RJ (2005) Age-related nuclear cataract-oxidation is the key. Exp Eye Res $\mathbf{8 0}, 709-725$.

34. Moeller SM, Taylor A, Tucker KL, et al. (2004) Overall adherence to the dietary guidelines for Americans is associated with reduced prevalence of early age-related nuclear lens opacities in women. J Nutr 134, 1812-1819

35. Mares JA, Voland R, Adler R, et al. (2010) Healthy diets and the subsequent prevalence of nuclear cataract in women. Arch Ophthalmol 128, 738-749.

36. Rautiainen S, Lindblad BE, Morgenstern R, et al. (2014) Total antioxidant capacity of the diet and risk of age-related cataract: a population-based prospective cohort of women. JAMA Ophthalmology 132, 247-252

37. Galea LM, Beck EJ, Probst YC, et al. (2017) Whole grain intake of Australians estimated from a cross-sectional analysis of dietary intake data from the 2011-13 Australian Health Survey. Public Health Nutr 20, 2166-2172.

38. Mann KD, Pearce MS, McKevith B, et al. (2015) Low whole grain intake in the UK: results from the National Diet and 
Nutrition Survey rolling programme 2008-11. Br J Nutr 113, $1643-1651$.

39. O'Neil CE, Nicklas TA, Zanovec M, et al. (2010) Wholegrain consumption is associated with diet quality and nutrient intake in adults: the National Health and Nutrition Examination Survey, 1999-2004. J Am Diet Assoc 110, 1461-1468.

40. Maras JE, Newby PK, Bakun PJ, et al. (2009) Whole grain intake: the Baltimore Longitudinal Study of Aging. J Food Compost Anal 22, 53-58.

41. Okarter N \& Liu RH (2010) Health benefits of whole grain phytochemicals. Crit Rev Food Sci Nutr 50, 193-208.

42. Brand-Miller JC, Stockmann K, Atkinson F, et al. (2009) Glycemic index, postprandial glycemia, and the shape of the curve in healthy subjects: analysis of a database of more than 1,000 foods. Am J Clin Nutr 89, 97-105.

43. Adom KK \& Liu RH (2002) Antioxidant activity of grains. J Agric Food Chem 50, 6182-6187.

44. Chatterjee A, Milton RC \& Thyle S (1982) Prevalence and aetiology of cataract in Punjab. Br J Ophthalmol 66, 35-42.

45. Townend BS, Townend ME, Flood V, et al. (2007) Dietary macronutrient intake and five-year incident cataract: the Blue Mountains Eye Study. Am J Ophthalmol 143, 932-939.

46. Mohan M, Sperduto RD, Angra SK, et al. (1989) India-US case-control study of age-related cataracts. Arch Ophthalmol 107, 670-676.

47. Messina V (2014) Nutritional and health benefits of dried beans. Am J Clin Nutr 100, Suppl. 1, 437S-442S.

48. Leske MC, Wu SY, Hyman L, et al. (1995) Biochemical factors in the lens opacities. Case-control study. The Lens
Opacities Case-Control Study Group. Arch Ophthalmol 113, 1113-1119.

49. Ramakrishnan S \& Sulochana KN (1993) Decrease in glycation of lens proteins by lysine and glycine by scavenging of glucose and possible mitigation of cataractogenesis. Exp Eye Res 57, 623-628.

50. Sulochana KN, Punitham R \& Ramakrishnan S (1998) Beneficial effect of lysine and amino acids on cataractogenesis in experimental diabetes through possible antiglycation of lens proteins. Exp Eye Res 67, 597-601.

51. Balfour JA \& Clissold SP (1990) Bendazac lysine. A review of its pharmacological properties and therapeutic potential in the management of cataracts. Drugs 39, 575-596.

52. Hockwin O, Laser H, De Gregorio M, et al. (1989) Bendazac lysine in selected types of human senile cataract. A long-term double-masked placebo-controlled clinical trial with multilinear densitometric image analysis of Scheimpflug photographs. Ophthalmic Res 21, 141-154.

53. Mudryj AN, Yu N \& Aukema HM (2014) Nutritional and health benefits of pulses. Appl Physiol Nutr Metab 39, 1197-1204.

54. Kuzniarz M, Mitchell P, Cumming RG, et al. (2001) Use of vitamin supplements and cataract: the Blue Mountains Eye Study. Am J Ophthalmol 132, 19-26.

55. Dalton SMC, Probst YC, Batterham MJ, et al. (2014) Compilation of an Australian database of manufactured and packaged food products containing wholegrain ingredients. J Food Compost Anal 36, 24-34.

56. Galea LM, Dalton SMC, Beck EJ, et al. (2016) Update of a database for estimation of whole grain content of foods in Australia. J Food Compost Anal 50, 23-29. 